\title{
The Thermal Conductivity of Carbon Nanotubes with Defects and Intramolecular Junctions
}

\author{
Qiaoli Zhou, ${ }^{1}$ Fanyan Meng, ${ }^{1}$ Zhuhong Liu, ${ }^{1}$ and Sanqiang Shi ${ }^{2}$ \\ ${ }^{1}$ Department of Physics, School of Mathematics and Physics, University of Science and Technology Beijing, Beijing 100083, China \\ ${ }^{2}$ Department of Mechanical Engineering, Hong Kong Polytechnic University, Hong Kong
}

Correspondence should be addressed to Fanyan Meng; meng7707@sas.ustb.edu.cn

Received 5 July 2013; Revised 11 November 2013; Accepted 15 November 2013

Academic Editor: Raymond Whitby

Copyright (C) 2013 Qiaoli Zhou et al. This is an open access article distributed under the Creative Commons Attribution License, which permits unrestricted use, distribution, and reproduction in any medium, provided the original work is properly cited.

\begin{abstract}
The thermal conductivity of various carbon nanotubes with defects or intramolecular junctions was studied using nonequilibrium molecular dynamics approach. The results show that the thermal conductivity of both armchair and zigzag carbon nanotubes increased with the decrease of the radius of the tube. The thermal conductivity of armchair tube is higher than that of zigzag tube when the radii of the two tubes are kept almost same. Discontinuities appear on the temperature profile along the tube axial at the region of IMJ, resulting in the large temperature gradient and thus lower thermal conductivity of $(n, n) /(m, 0)$ tube with one IMJ and $(m, 0) /(n, n) /(m, 0)$ tube with two IMJs. For the $(m, 0) /(n, n) /(m, 0)$ tube with two IMJs, phonon mean free path of the middle $(n, n)$ tube is much smaller than that of the isolate $(n, n)$ tube.
\end{abstract}

\section{Introduction}

As the sizes of electronic and mechanical devices are decreased to the micron and nanometre level, there is a great interest in materials that conduct heat efficiently, thus preventing structural damage $[1,2]$. Carbon allotropes and their derivatives occupy a unique place in terms of their ability to conduct heat [3], especially the carbon nanotubes (CNTs). As a typical one-dimensional nanostructure material, CNTs possessing the unique mechanical, thermodynamic, and electronic properties [4-7] have received considerable attention, since the discovery of multiwalled carbon nanotubes by Iijima [8] in 1991 and single-walled nanotubes by Iijima and Ichihashi [9] and Bethune et al. [10] in 1993. The high thermal conductivities of carbon nanotubes are measured experimentally [11-15] and theoretically $[1,2,16-19]$, revealing that they are promising building blocks for advanced technologies in the future [20].

The previous experiments $[13,21-25]$ have been conducted to measure the thermal properties of millimeter-sized CNT mats, graphene and packed carbon nanofibers, which may help us a little further to understand the thermal properties of these materials. Up to now, there are many reports on thermal conductivity of isolated carbon nanotubes and more emphasis on a good medium for the perfect carbon nanotubes. However, there has been little systematic investigation on the thermal conductivity of nanotubes with defects. Che et al. [1] compared the effects of vacancies with that of StoneWales (SW) defects on the thermal conductivity of CNTs. The calculated results of Cummings et al. [26] demonstrate that a two-atom-vacancy defect is similar to one Y-junction. The thermal conductivity of CNTs with only $0.25 \%$ vacancies decreases $60 \%$ at $300 \mathrm{~K}$, which is predicted by Bi et al. [27]. Nevertheless, Kondo et al. [20] reported that the thermal conductivity of carbon nanotubes decreases rapidly down to $25 \%$ with only $1 \%$ vacancy defects. Meanwhile, Kondo et al. [20] found that the thermal resistance of the 5-6 defects is smaller than that of the vacancy defects. In our previous work, we studied that the thermal conductivity of the nanotube with junctions was $20-80 \%$ less than that of a straight nanotube depending on temperature [28]. Other defects such as isotropic impurities [27], $s p^{3}$-hybridized defect [29], chemisorptions [30], and oxidation on the thermal conductivity of SWCNTs were studied.

In the following, we investigate the thermal conductivities of various carbon nanotubes with one or two intramolecular junctions (IMJs) using molecular dynamics (MD) approach 


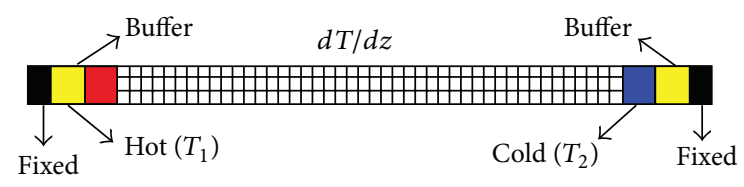

FIGURE 1: Molecular dynamics setup for calculating the thermal conductivity of a straight carbon nanotube (colored). A temperature differential $\pm 50 \mathrm{~K}$ is applied at the hot and the cold reservoirs.

and discuss the effect of Stone-Wales and vacancy on the thermal conductivity. It is concluded that the chiralities, size, and defects play a role in the thermal properties of SWCNTs. The details for the model and results are discussed in Sections 2 and 3 , respectively.

\section{Methodology}

In this paper, MD scheme is chosen to simulate the lattice thermal conductivity. The setup model for the MD approach similar with Cummings et al. [26] is shown in Figure 1. The straight SWCNT is split into a series of equal "slabs" of atoms. Both ends of the tube are fixed in space in order to prevent tube drift and oscillations during the simulation, labeled "Fixed" in Figure 1. The tube is in contact with two thermal reservoirs at temperatures $T_{1}$ and $T_{2}$ at two end regions, respectively, indicated by "Hot" and "Cold." The length of each thermal reservoir is about $0.25 \mathrm{~nm}$. The temperatures of the hot and cold reservoirs are regulated by a scaling of the velocities of the atoms within the thermal reservoirs. The buffer regions are introduced between the fixed regions and thermal reservoirs, labeled "Buffer" to neglect edge effects. The temperature for the buffer atoms is regulated by the Langevin dynamics approach using more realistic friction and random forces. The thermal conductivity $\lambda$ along the tube axis, taken here as the $z$ axis, is the quotient of the heat flux density $J$ and the temperature gradient $d T / d z$ based on the Fourier's law:

$$
\lambda=-\frac{J}{d T / d z}, \quad J=\frac{1}{A} \frac{d \mathrm{Q}}{d t},
$$

where $A$ is the cross-sectional annular ring area of the tube and $d Q$ is the energy transmitted across the area $A$ in the time interval $d t$. The heat flux density $J$ is calculated by taking an average of the net kinetic energy added at each previous time step after the system arrives at the steady state. The average temperature gradient can be obtained by applying a linear least-squares fitting to the temperatures of these slabs between the thermal reservoirs. The tubes were run at $300 \mathrm{~K}$ about $0.5 \mathrm{~ns}$ for homogenization, and then the temperature differential $\pm 50 \mathrm{~K}$ is applied at the hot and cold reservoirs. A total time of $1.5 \mathrm{~ns}$ was used for the measurement of temperature gradient. A nonequilibrium molecular-dynamics (MD) simulation is performed to calculate $\lambda$ using reactive empirical bond order potential [31]. Hamilton's classical equation of motion is solved using a predictor-corrector algorithm with a fixed time step of $0.5 \mathrm{fs}$. A ring area of van der Waals thickness of $3.4 \AA$ was employed as the cross-section area. Many processes involved in the thermal conductivity such as boundary

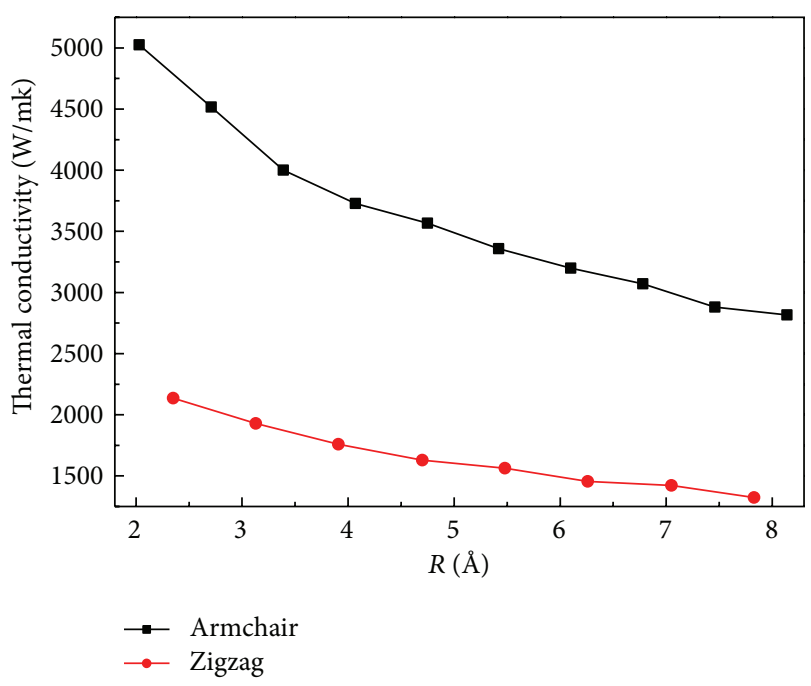

FIGURE 2: The thermal conductivity of $(m, 0)$ tubes and $(n, n)$ tubes dependent on the radius of the SWCNTs (colored).

scattering, crystal imperfections, surface effects, and isotope effects are included in the MD simulations. In this paper, we simulate the thermal conductivities of both armchair and zigzag SWCNTs, the SWCNT with one intramolecular junction, and two intramolecular junctions.

\section{Results and Discussions}

3.1. The Effects of Radius and Chirality on the Thermal Conductivity. As we know, the thermal conductivity of the tube is greatly dependent on the length of the tube, and we have discussed this in our previous work [28]. In our present simulations, the length for the calculation of temperature gradient $d T / d z$ is about $17 \mathrm{~nm}$ for armchair tubes and zigzag tubes to save the simulation time. This length is used just for the qualitative comparison of pristine tube and tube with defects or intramolecular junctions, but not for absolute values of thermal conductivity. To discuss the effects of radius and chirality on the thermal conductivity of the tube, $(m, 0)$ and $(n, n)$ tubes are investigated, and the results are shown in Figure 2.

The thermal conductivity of CNTs decreases with the increasing of radius, as depicted in Figure 2. The results demonstrated that the thermal conductivity of armchair tube is more sensitive to the radius than that of zigzag tube. When the radii are kept almost the same, the thermal conductivity of armchair CNTs is much higher than that of zigzag CNTs. It is known that in the model where the phonon contribution is only considered, the thermal conductivity is proportional to $C v l \sim C v^{2} \tau \sim C v D$, in which $C$ is the heat capacity per volume, $v$ is the speed of sound, $l$ is the mean free path, $\tau$ is the phonon lifetime, and $D$ is the nanostructure or grain size $[3,32]$. The speed of sound $v$ in the SWCNT is proportional to $\sqrt{Y / \rho}$, where $Y$ is Young's modulus of the tube and $\rho$ is the linear density of the tube. Young's modulus of armchair tube is higher than that of zigzag tube [33], and the linear density of armchair one is lower than that of zigzag one. Both length and radius are the same, so $D$ is also the same. In nanostructures 


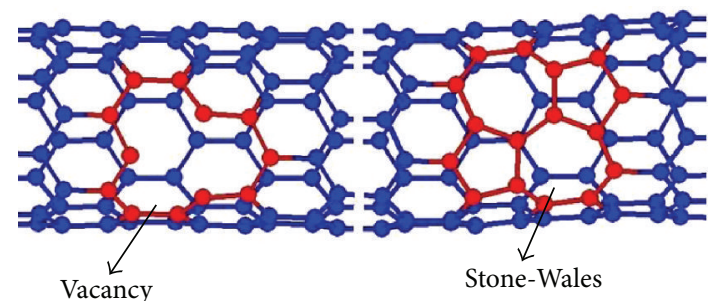

(a)

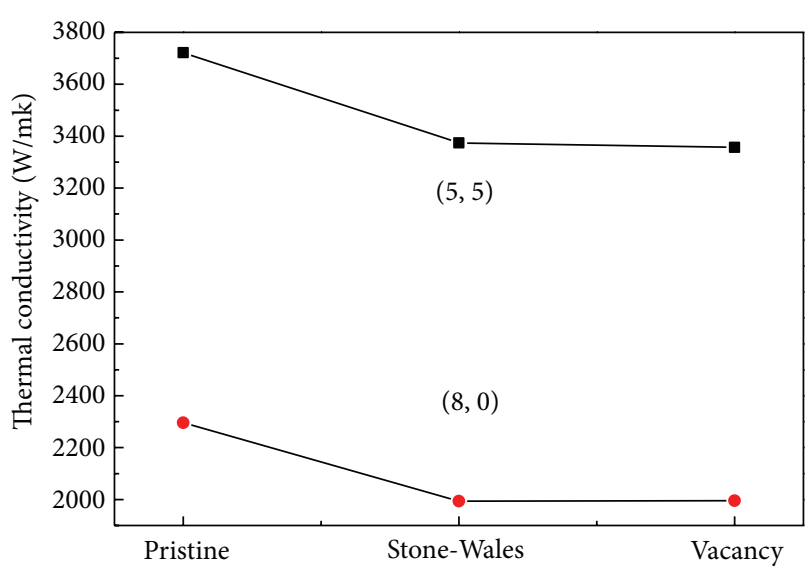

(b)

FIgURE 3: (a) The structure of the defective SWCNTs; (b) the thermal conductivity of the defective SWCNTs (colored).

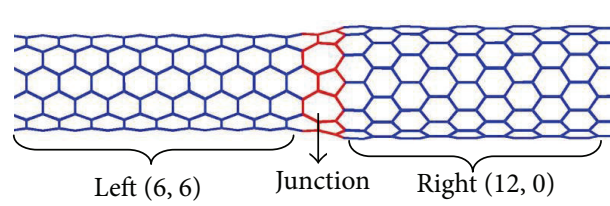

(a)

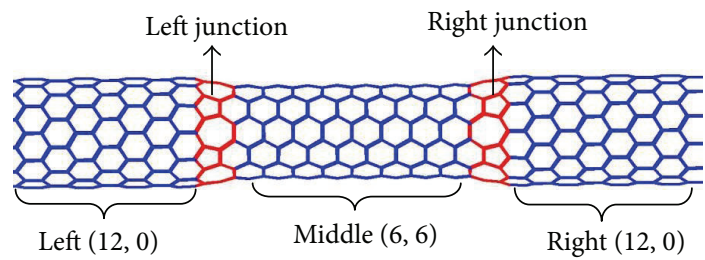

(b)

FIGURE 4: The atomic configuration of SWCNTs with one IMJ (a) and that with two IMJs (b) (colored).

with $D \ll l$, phonon dispersion can undergo modifications owing to confinement resulting in changes in $v$ and more complicated size dependence $[3,34-36]$, which result in the higher thermal conductivity of armchair tube.

And as the radius increases, the difference in thermal conductivity between armchair tube and zigzag tube decreases. The results are consistent with other calculated results [37, 38]. It is suggested that the thermal conductivity is not sensitive to the chirality when the diameter of the SWCNT becomes very large.

3.2. The Effects of Stone-Wales and Vacancy on the Thermal Conductivity. In order to understand the effects of the intramolecular junctions on the thermal conductivity, we simulate the thermal conductivities of the tubes with a StoneWales (SW) defect or a vacancy. The atomic configuration and the thermal conductivity of the defective tubes are shown in Figure 3. Discontinuities in the temperature profile across the defects have been observed in [28]. Conformational defects can reduce the thermal conductivity significantly. These defects act as the high resistance to heat flow, resulting in the relatively large temperature gradient at the region near the defects (SW defect or vacancy) in both $(5,5)$ and $(8$, $0)$ tubes. The high resistance to heat flow contributes to the lower values for the thermal conductivity of the defective tube, as clearly shown in Figure 3(b). It is also shown that the thermal conductivity of the tube with a vacancy is lower than that with a SW defect, since a SW defect does not change the basic bonding characteristic and causes much less overall structural deformation. The calculation result is consistent with [1]. Therefore, the thermal conductivity remarkably decreases with the introduction of vacancy.

3.3. The Thermal Conductivity of CNTS with IMJs. The results above indicate that the decrease in the thermal conductivity associates with the presence of defects in the crystal lattice, since these defects act as additional phonon scattering centers. In the IMJs model under investigation in this work, the lattice defects are present in the form of many pentagonheptagon pairs (5/7 pairs) at the junction point, since $(n, m)$ and $(n-1, m+1)$ tubes of different chirality can be connected by 5/7 pairs [26]. Ouyang et al. [39] have studied different tubes connected with an intramolecular junction. In our work, we investigate various tubes connected with one or two intramolecular junctions, to understand the effects of chirality, length and defective structure on the thermal conductivity. The atomic structures for one intramolecular junction in $(n, n) /(m, 0)$ tube and for two intramolecular junctions in $(m, 0) /(n, n) /(m, 0)$ tube are shown in Figures $4(\mathrm{a})$ and $4(\mathrm{~b})$, respectively. In the one intramolecular junction model, the left is $(6,6)$ or $(n, n)$ armchair tube with length of $61.5 \AA$, and the right is $(12,0)$ or $(m, 0)$ zigzag tube with length of $106.5 \AA$. The junction that connected the tubes consists of $n 5 / 7$ pairs. In the two intramolecular junctions model, both left and right are $(12,0)$ or $(m, 0)$ zigzag tubes with length of $85.2 \AA$, and the middle is the armchair $(6,6)$ or $(n, n)$ tube, whose length is 24.6 $\AA$. Both intramolecular junctions consist of $n 5 / 7$ pairs. 


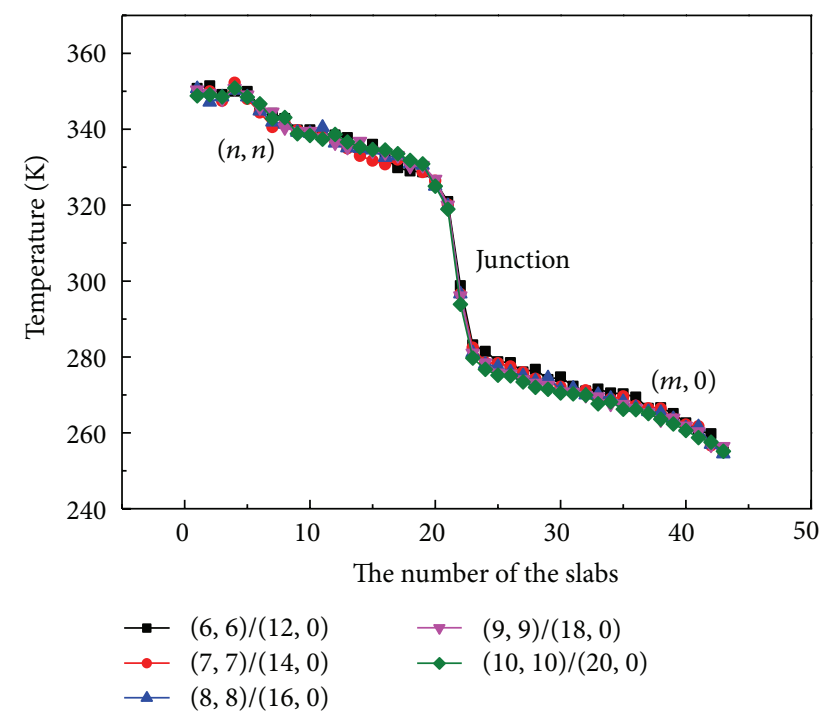

FIGURE 5: The temperature profile along the straight $(n, n) /(m, 0)$ tube with one IMJ (colored).

3.3.1. The Thermal Conductivity of Various $(n, n) /(m, 0)$ Tubes with One IMJ. Figure 5 shows that there are sharp discontinuities in the temperature profiles along $z$ axis at the local region of all the IMJs. In each of these cases, the jump in the temperature profile seems to be associated with the presence of discontinuities [26]. It is seen from Figure 5 that there is a sudden fall of temperature of various $(n, n) /(m, 0)$ tubes with one IMJ at the IMJs regions. However, the temperature profiles along $z$ axis of both left and right decrease slowly. As we know, the interfaces are the phonon scattering centers acting as the high resistance to heat flow. So, the IMJs have much higher temperature gradients than both pristine tube and defective tube studied above, because the IMJs have more defects acting as larger phonon scattering centers. It is clearly shown in Figure 6 that the thermal conductivity of the IMJs greatly decreases to compare with those of the left and right tubes. The drop in the thermal conductivity of the IMJ is about $2000 \mathrm{~W} / \mathrm{m} / \mathrm{K}$ relative to those of both left and right tubes, while the thermal conductivity of both left and right tubes is much higher than that of isolate $(n, n)$ or $(m, 0)$ tubes under the same length.

3.3.2. The Thermal Conductivity of $(m, 0) /(n, n) /(m, 0)$ Tube with Two IMJs. The temperature profile along the straight $(m, 0) /(n, n) /(m, 0)$ tube with two IMJs is shown in Figure 7. Two jumps appear in the temperature profile at the two IMJs regions, since scattering centers exist. The distribution feature and the fluctuation tendency of the temperature of $(m, 0) /(n, n) /(m, 0)$ tubes with two IMJs are all very similar. These defects act as additional scattering centers and result in a region of large temperature gradient, which translates to a reduction in the thermal conductivity of the crystal [26]. The thermal conductivities of each part along the $(m, 0) /(n, n) /(m, 0)$ tube are shown in Figure 8. The thermal conductivity of various IMJs does not appear to be much different and is about $300 \mathrm{~W} / \mathrm{m} / \mathrm{K}$. The thermal conductivities

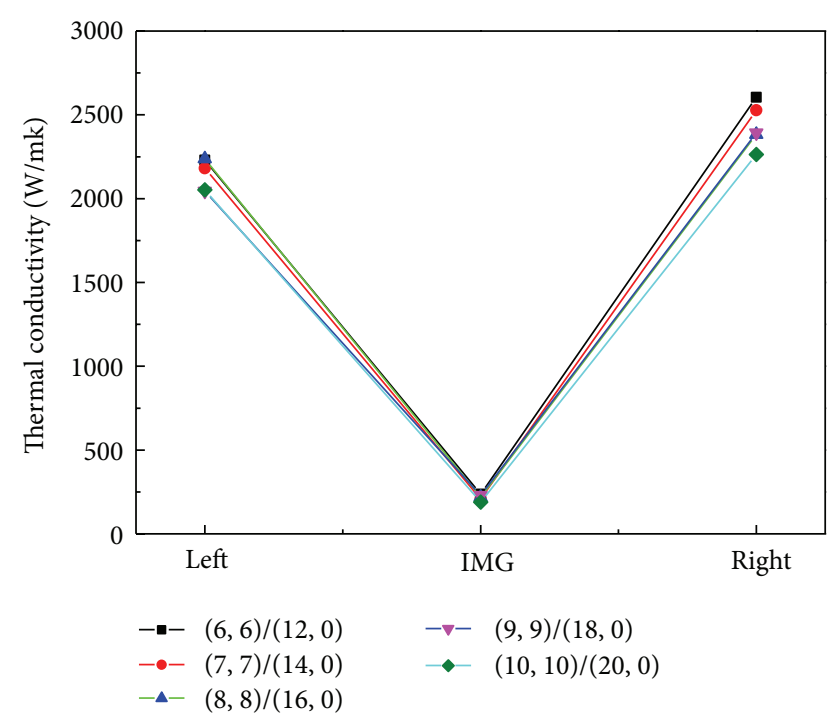

FIGURE 6: The thermal conductivity of different parts of $(n, n) /(m, 0)$ tubes with one IMJ (colored).

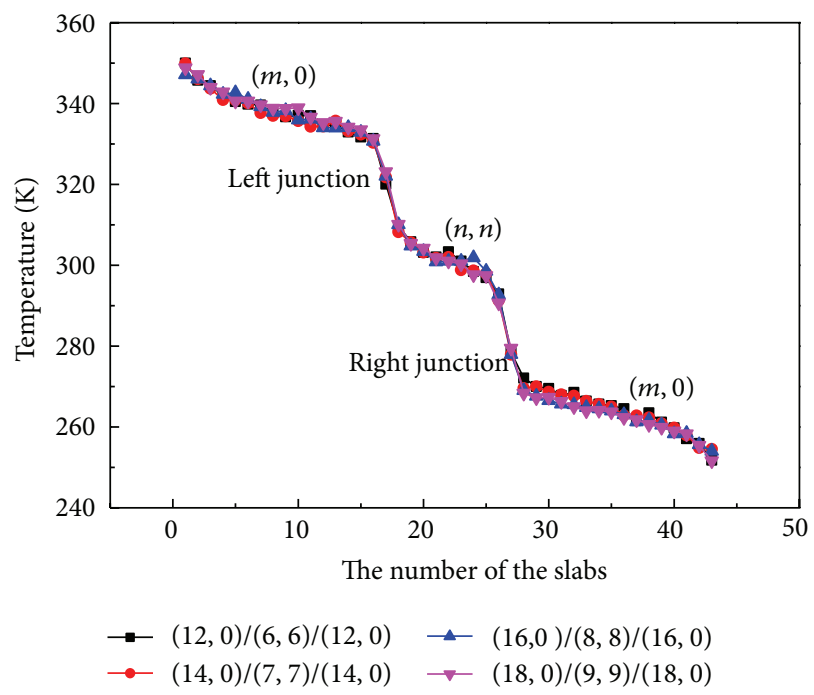

Figure 7: The temperature profile of $(m, 0) /(n, n) /(m, 0)$ tube with two IMJs (colored).

of the left and right $(m, 0)$ tubes is different from each other owing to difference of the temperature areas. The thermal conductivity of the middle $(n, n)$ tube does not follow the normal law that the thermal conductivity of tubes are decreased with increasing radius. Both defects at the two IMJs regions and the size confinement of the middle tube influence the thermal conductivity of the $(m, 0) /(n, n) /(m, 0)$ tube.

3.3.3. Effects of the Length of the Middle Tube on the Thermal Conductivity. To find out the effects of the length of the middle tube on the thermal conductivity of the $(m, 0) /(n, n) /(m, 0)$ tube, we studied $(12,0) /(6,6) /(12,0)$ tube with various size of the middle $(6,6)$ tube. Different amounts of unit cell of $(6,6)$ tube, are considered while the right 


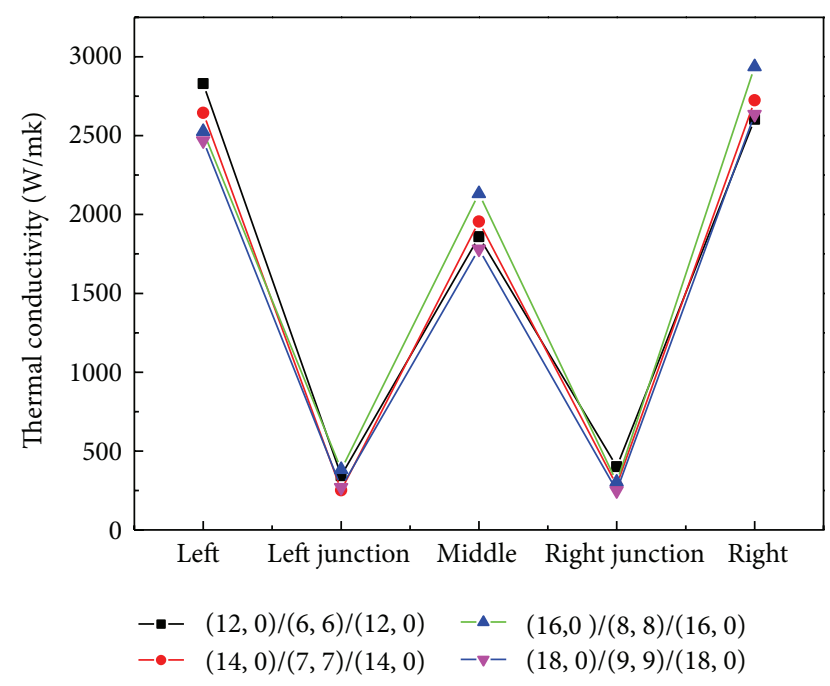

FIgURE 8: The thermal conductivity of different parts of $(m, 0) /(n, n) /(m, 0)$ tube with two IMJs (colored).

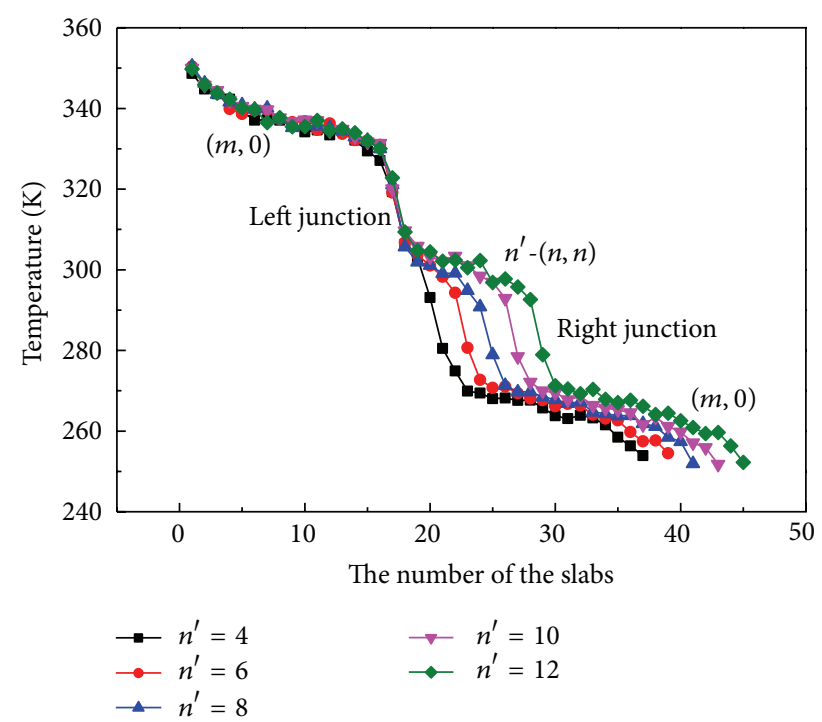

FIgURE 9: The temperature profile of $(m, 0) / n^{\prime}-(n, n) /(m, 0)$ tubes with two IMJs (colored).

and left $(12,0)$ tube remained unchanged. $n^{\prime}$ is used here to indicate the number of unit cells of $(6,6)$ tube, varying from 4 to 12 ; that is, the length of $(6,6)$ tube ranges from $9.84 \AA$ to $29.52 \AA$. So, the middle $(6,6)$ tube with different size is expressed as $n^{\prime}-(6,6)$ tube for brief and clear. The results of $(12,0) / n^{\prime}-(6,6) /(12,0)$ tubes are shown in Figure 9. The temperature profiles of left and right $(12,0)$ tube barely change, and the slops at the left IMJ and right IMJ are almost the same. The temperature gradients at the middle $n^{\prime}-(6,6)$ tube are quite different due to the different values of $n^{\prime}$, and the absolute temperature gradient is getting smaller with increase of $n^{\prime}$. The thermal conductivity of each part of the $(12,0) / n^{\prime}$ $(6,6) /(12,0)$ tube is shown in Figure 10. It is worth noting that the thermal conductivity of middle $n^{\prime}-(6,6)$ tube is

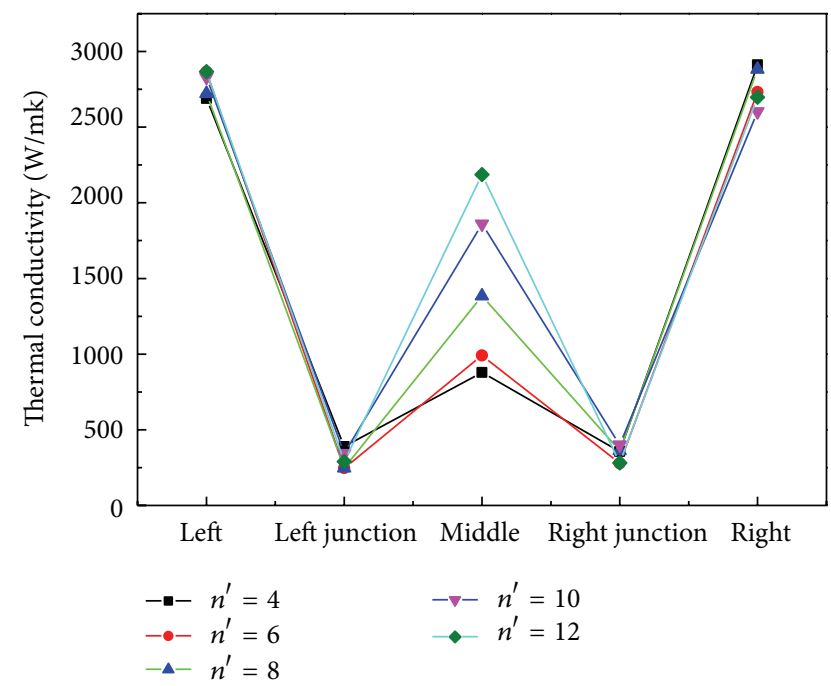

FIGURE 10: The thermal conductivity of different parts of $(m, 0) / n^{\prime}$ $(n, n) /(m, 0)$ tubes (colored).

within the range of $800 \sim 2200 \mathrm{~W} / \mathrm{m} / \mathrm{K}$, which is much higher than that of isolate $n^{\prime}-(6,6)$ tube (less than $400 \mathrm{~W} / \mathrm{m} / \mathrm{K}$ ). As we know the interfaces are the phonon scattering centers acting as the high resistance to heat flow. The interfaces for the isolate $n^{\prime}-(6,6)$ tube are the hot and cold thermal reservoirs, while for middle $n^{\prime}-(6,6)$ tube they are the left and right IMJs. The quite large difference in the thermal conductivity between the middle $n^{\prime}-(6,6)$ tubes and the isolate $n^{\prime}-(6,6)$ tubes can be explained that the scattering between thermal reservoirs is greatly stronger than that between IMJs. Therefore, the phonon-mean-free-path of the isolate tube is much longer than that of middle tube. The thermal conductivity of the middle $(6,6)$ tube is more than $2000 \mathrm{~W} / \mathrm{m} / \mathrm{K}$ when it is $\sim 3 \mathrm{~nm}$ long (with $n^{\prime}$ is 12) and is increased with the increasing of the length of the tubes within a certain range, which is identical with $[1,28,40,41]$. Therefore, the IMJs play a major role in the thermal conductivity of the $(m, 0) /(n, n) /(m, 0)$ tube with two IMJs.

\section{Conclusions}

To conclude, the thermal conductivity of both of armchair and zigzag carbon nanotubes increased with the decrease of the radius of the tube. The thermal conductivity of armchair tube is higher than that of zigzag tube when the radii of the two tubes are kept almost same. Great discontinuities appear on the temperature profile along the tube axial at the region of IMJ, resulting in the large temperature gradient and thus lower thermal conductivity of $(n, n) /(m, 0)$ tube with one IMJ and $(m, 0) /(n, n) /(m, 0)$ tube with two IMJs. For the $(m, 0) /(n, n) /(m, 0)$ tube with two IMJs, phonon mean free path of the middle $(n, n)$ tube is much smaller than that of the isolate $(n, n)$ tube. When the length of the middle $(n, n)$ tube is more than $3 \mathrm{~nm}$, the thermal conductivity is only affected by the IMJs. 


\section{Acknowledgments}

This work was supported by the National Natural Science Foundation of China (Grant no. 50902009), Fundamental Research Project of Ministry of Science and Technology (Grant no. 2011CB606406), and the Fundamental Research Funds for the University of Science and Technology Beijing (Grant no. 06108105).

\section{References}

[1] J. Che, T. Çaǧin, and W. A. Goddard III, "Thermal conductivity of carbon nanotubes," Nanotechnology, vol. 11, no. 2, pp. 65-69, 2000.

[2] S. Berber, Y.-K. Kwon, and D. Tománek, "Unusually high thermal conductivity of carbon nanotubes," Physical Review Letters, vol. 84, no. 20, pp. 4613-4616, 2000.

[3] A. A. Balandin, "Thermal properties of graphene and nanostructured carbon materials," Nature Materials, vol. 10, no. 8, pp. 569-581, 2011.

[4] L. Chico, V. H. Crespi, L. X. Benedict, S. G. Louie, and M. L. Cohen, "Pure carbon nanoscale devices: nanotube heterojunctions," Physical Review Letters, vol. 76, no. 6, pp. 971-974, 1996.

[5] L. Langer, V. Bayot, E. Grivei et al., "Quantum transport in a multiwalled carbon nanotube," Physical Review Letters, vol. 76, no. 3, pp. 479-482, 1996.

[6] T. W. Ebbesen, H. J. Lezec, H. Hiura, J. W. Bennett, H. F. Ghaemi, and T. Thio, "Electrical conductivity of individual carbon nanotubes," Nature, vol. 382, no. 6586, pp. 54-56, 1996.

[7] T. Pichler, M. Knupfer, M. S. Golden, J. Fink, A. Rinzler, and R. E. Smalley, "Localized and delocalized electronic states in single-wall carbon nanotubes," Physical Review Letters, vol. 80, no. 21, pp. 4729-4732, 1998.

[8] S. Iijima, "Helical microtubules of graphitic carbon," Nature, vol. 354, no. 6348, pp. 56-58, 1991.

[9] S. Iijima and T. Ichihashi, "Single-shell carbon nanotubes of 1nm diameter," Nature, vol. 363, no. 6430, pp. 603-605, 1993.

[10] D. S. Bethune, C. H. Kiang, M. S. de Vries et al., "Cobaltcatalysed growth of carbon nanotubes with single-atomic-layer walls," Nature, vol. 363, no. 6430, pp. 605-607, 1993.

[11] Y. A. Kim, S. Kamio, T. Tajiri et al., "Enhanced thermal conductivity of carbon fiber/phenolic resin composites by the introduction of carbon nanotubes," Applied Physics Letters, vol. 90, no. 9, Article ID 093125, 2007.

[12] E. Pop, D. Mann, Q. Wang, K. Goodson, and H. Dai, “Thermal conductance of an individual single-wall carbon nanotube above room temperature," Nano Letters, vol. 6, no. 1, pp. 96-100, 2006.

[13] J. Hone, M. Whitney, C. Piskoti, and A. Zettl, “Thermal conductivity of single-walled carbon nanotubes," Physical Review B, vol. 59, no. 4, pp. R2514-R2516, 1999.

[14] M. Fujii, X. Zhang, H. Xie et al., "Measuring the thermal conductivity of a single carbon nanotube," Physical Review Letters, vol. 95, no. 6, Article ID 065502, 2005.

[15] P. Kim, L. Shi, A. Majumdar, and P. L. McEuen, "Thermal transport measurements of individual multiwalled nanotubes," Physical Review Letters, vol. 87, no. 21, Article ID 215502, 4 pages, 2001.

[16] J. F. Moreland, J. B. Freund, and G. Chen, “The disparate thermal conductivity of carbon nanotubes and diamond nanowires studied by atomistic simulation," Microscale Thermophysical Engineering, vol. 8, no. 1, pp. 61-69, 2004.

[17] M. Alaghemandi, E. Algaer, M. C. Böhm, and F. Müller-Plathe, "The thermal conductivity and thermal rectification of carbon nanotubes studied using reverse non-equilibrium molecular dynamics simulations," Nanotechnology, vol. 20, no. 11, Article ID 115704, 2009.

[18] M. A. Osman and D. Srivastava, "Temperature dependence of the thermal conductivity of single-wall carbon nanotubes," Nanotechnology, vol. 12, no. 1, pp. 21-24, 2001.

[19] J. A. Thomas, R. M. Iutzi, and A. J. H. McGaughey, “Thermal conductivity and phonon transport in empty and water-filled carbon nanotubes," Physical Review B, vol. 81, no. 4, Article ID 045413, 2010.

[20] N. Kondo, T. Yamamoto, and K. Watanabe, "Moleculardynamics simulations of thermal transport in carbon nanotubes with structural defects," e-Journal of Surface Science and Nanotechnology, vol. 4, pp. 239-243, 2006.

[21] J. Hone, M. C. Llaguno, M. J. Biercuk et al., "Thermal properties of carbon nanotubes and nanotube-based materials," Applied Physics A, vol. 74, no. 3, pp. 339-343, 2002.

[22] J. Hone, M. C. Llaguno, N. M. Nemes et al., "Electrical and thermal transport properties of magnetically aligned single wall carbon nanotube films," Applied Physics Letters, vol. 77, no. 5, pp. 666-668, 2000.

[23] W. Yi, L. Lu, Z. Dian-lin, Z. W. Pan, and S. S. Xie, "Linear specific heat of carbon nanotubes," Physical Review B, vol. 59, no. 14, pp. R9015-R9018, 1999.

[24] D. L. Nika and A. A. Balandin, "Two-dimensional phonon transport in graphene," Journal of Physics, vol. 24, no. 23, Article ID 233203, 2012.

[25] R. Gulotty, M. Castellino, P. Jagdale et al., "Effects of functionalization on thermal properties of single-wall and multiwall carbon nanotube-polymer nanocomposites," ACS Nano. In press.

[26] A. Cummings, M. Osman, D. Srivastava, and M. Menon, "Thermal conductivity of Y-junction carbon nanotubes," Physical Review B, vol. 70, no. 11, Article ID 115405, 6 pages, 2004.

[27] K. Bi, Y. Chen, J. Yang, and M. Chen, "Molecular dynamics simulation of thermal conductivity of single-wall carbon nanotubes with different structures," Journal of Southeast University (Natural Science Edition), vol. 36, no. 3, pp. 420-422, 2006.

[28] F. Y. Meng, S. Ogata, D. S. Xu et al., "Thermal conductivity of an ultrathin carbon nanotube with an X-shaped junction," Physical Review B, vol. 75, no. 20, Article ID 205403, 6 pages, 2007.

[29] H. Fan, K. Zhang, and M. M. F. Yuen, "Effect of defects on thermal performance of carbon nanotube investigated by molecular dynamics simulation," in Proceedings of the International Conference on Electronic Materials and Packaging (EMAP'06), IEEE, Kowloon, China, December 2006.

[30] C. W. Padgett and D. W. Brenner, "Influence of chemisorption on the thermal conductivity of single-wall carbon nanotubes," Nano Letters, vol. 4, no. 6, pp. 1051-1053, 2004.

[31] D. W. Brenner, O. A. Shenderova, J. A. Harrison, S. J. Stuart, B. $\mathrm{Ni}$, and S. B. Sinnott, "A second-generation reactive empirical bond order (REBO) potential energy expression for hydrocarbons," Journal of Physics Condensed Matter, vol. 14, no. 4, pp. 783-802, 2002.

[32] A. A. Balandin and D. L. Nika, "Phononics in low-dimensional materials," Materials Today, vol. 15, no. 6, pp. 266-275, 2012. 
[33] Y. D. Wu, W. F. Zhong, and A. Y. T. Leung, "Study on the Young's modulus of single-walled carbon nanotubes via energy approach," Journal of Materials Science and Engineering, vol. 24, no. 1, pp. 85-88, 2006.

[34] A. Balandin and K. L. Wang, "Effect of phonon confinement on the thermoelectric figure of merit of quantum wells," Journal of Applied Physics, vol. 84, no. 11, pp. 6149-6153, 1998.

[35] A. A. Balandin, E. P. Pokatilov, and D. L. Nika, "Phonon engineering in hetero- and nanostructures," Journal of Nanoelectronics and Optoelectronics, vol. 2, no. 2, pp. 140-170, 2007.

[36] A. A. Balandin, "Nanophononics: phonon engineering in nanostructures and nanodevices," Journal of Nanoscience and Nanotechnology, vol. 5, no. 7, pp. 1015-1022, 2005.

[37] L. Lindsay, D. A. Broido, and N. Mingo, "Diameter dependence of carbon nanotube thermal conductivity and extension to the graphene limit," Physical Review B, vol. 82, no. 16, Article ID 161402, 2010.

[38] C. Yu, L. Shi, Z. Yao, D. Li, and A. Majumdar, “Thermal conductance and thermopower of an individual single-wall carbon nanotube," Nano Letters, vol. 5, no. 9, pp. 1842-1846, 2005.

[39] M. Ouyang, J. L. Huang, C. L. Cheung et al., "Energy gaps in, "metallic" single-walled carbon nanotubes," Science, vol. 292, no. 5517, pp. 702-705, 2001.

[40] A. N. Volkov and L. V. Zhigilei, "Heat conduction in carbon nanotube materials: Strong effect of intrinsic thermal conductivity of carbon nanotubes," Applied Physics Letters, vol. 101, no. 4, Article ID 043113, 2012.

[41] N. Mingo and D. A. Broido, "Length dependence of carbon nanotube thermal conductivity and the 'problem of long waves," Nano Letters, vol. 5, no. 7, pp. 1221-1225, 2005. 

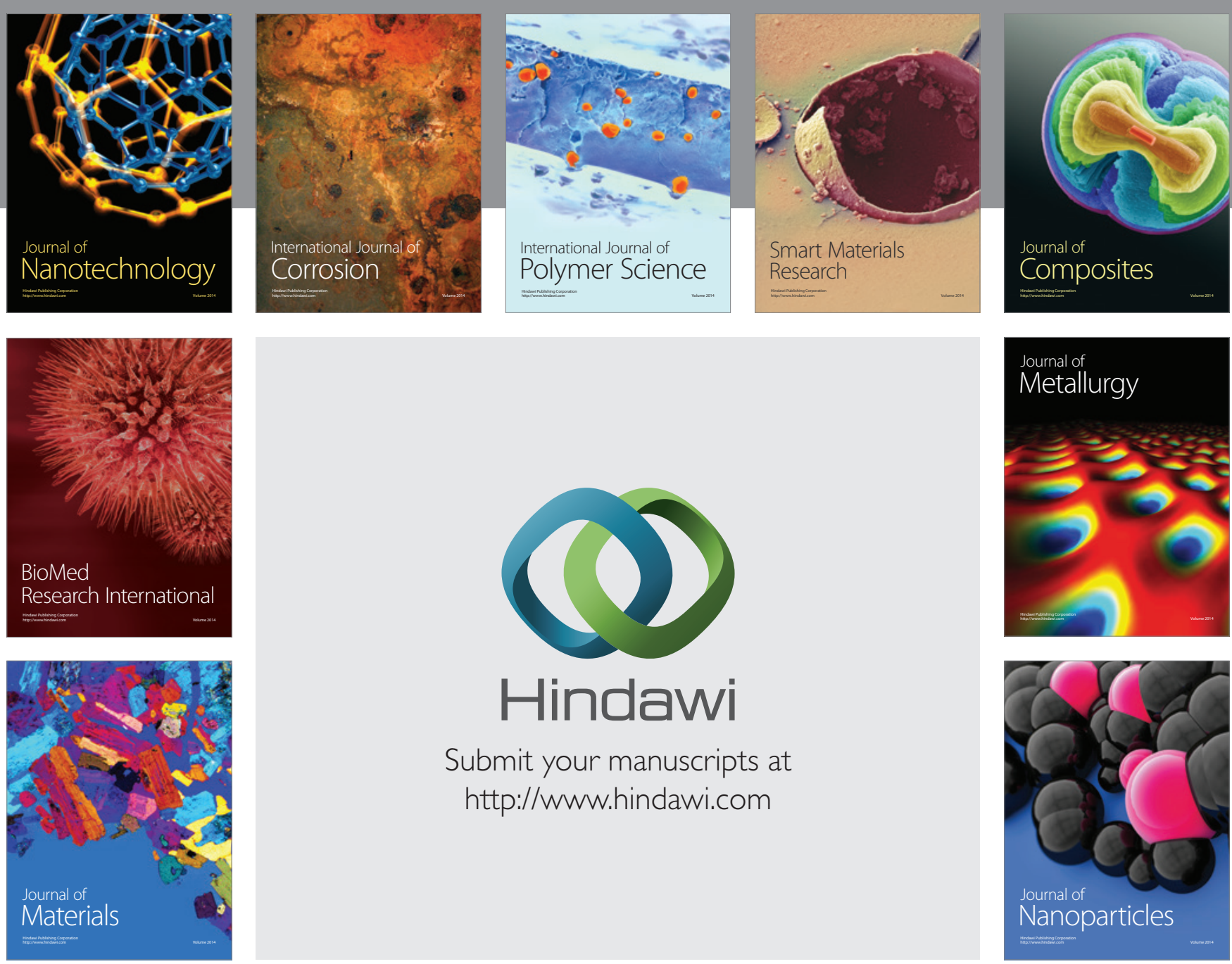

Submit your manuscripts at http://www.hindawi.com
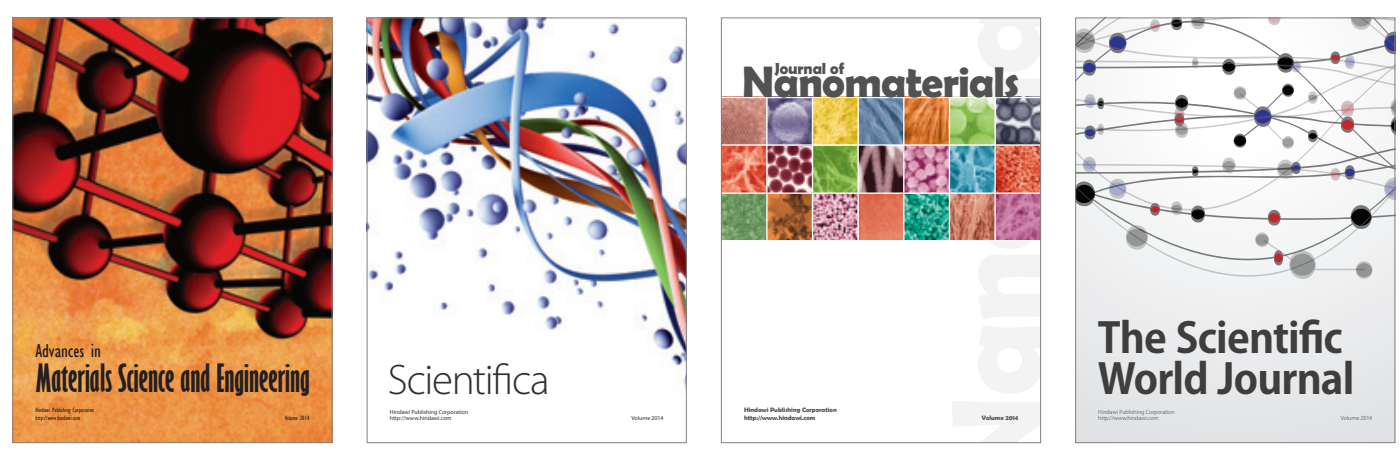

\section{The Scientific World Journal}
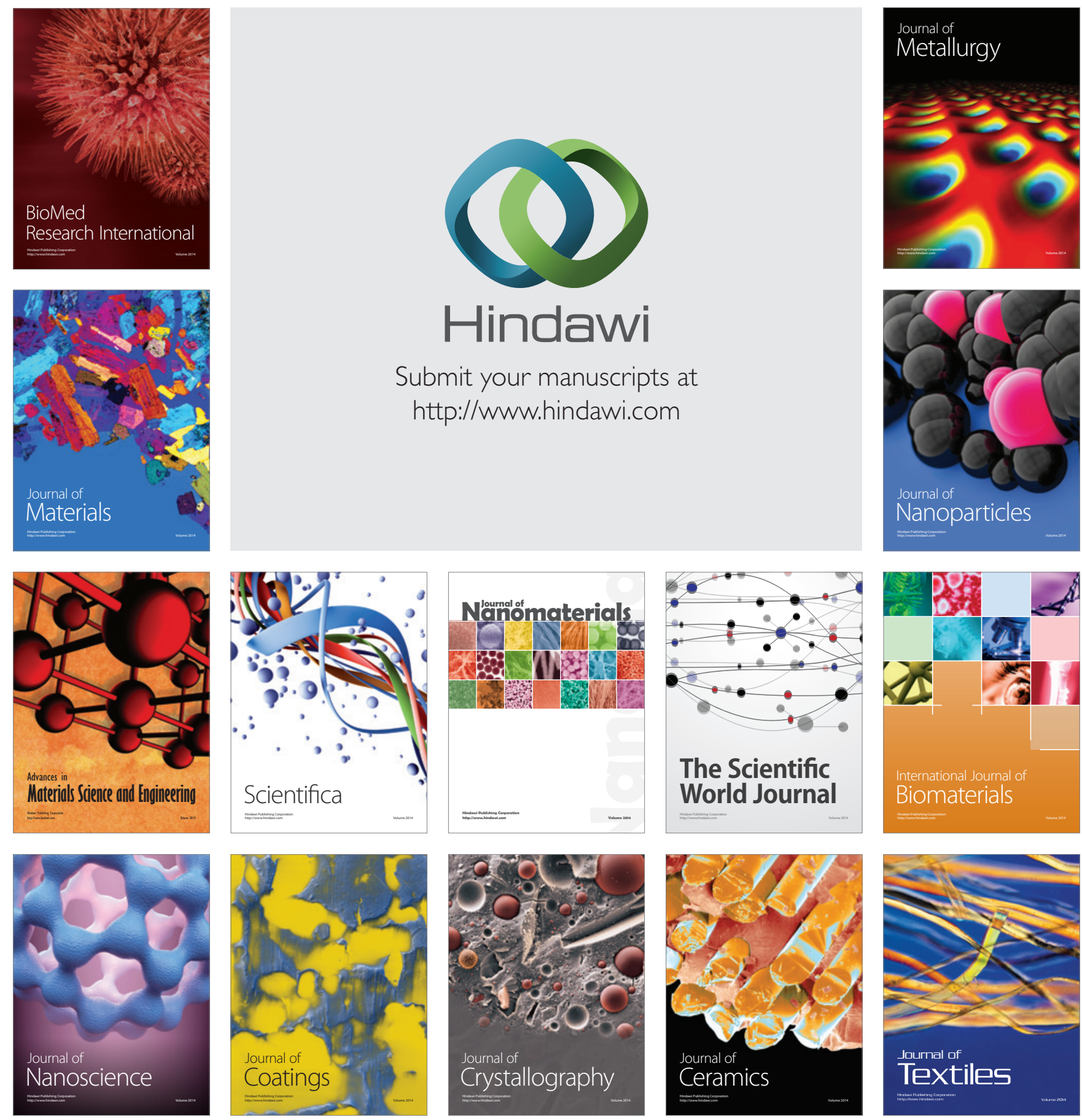